\title{
Congenital Uterine Anomaly: Successful Pregnancy Outcome in Bicornuate Uterus
}

\author{
Tayade Surekha A ${ }^{1}$, Naina Kumar ${ }^{2}$ \\ ${ }^{1}$ Professor, 2 Assistant Professor Departments of Obstetrics and Gynecology, Mahatma Gandhi Institute of \\ Medical Sciences, Seagram
}

\begin{abstract}
We report a case of persistent breech presentation in a primigravida with bicornuate uterus that was initially diagnosed by early ultrasound scan. Persistent breech presentation later in the pregnancy necessitated an elective caesarean section at term. The diagnosis was confirmed intraoperatively by exteriorizing the uterus. The literature regarding bicornuate uterus was reviewed.
\end{abstract}

Keywords-Bicornuate uterus, Persistent breech, Mullerian ducts.

\section{INTRODUCTION}

Congenital abnormalities of the uterus including bicornuate uterus are rare but known to be associated with infertility, spontaneous miscarriages, intrauterine growth restriction, preterm deliveries, preterm prelabour rupture of membranes, breech presentation and increased rate of caesarean delivery [1-3]. However, normal reproductive performance has also been documented in association with them [4]. The prevalence of uterine anomalies in the general population is about 0.5\% [5]. Imo and Sunday-Adeoye found a prevalence of $0.8 \%$ in patients who had hysterosalpingography in the radiology unit of Ebonyi State University Teaching Hospital between 2002 and 2005 [6] . The true incidence of these congenital anomalies might be understated as many of the women with the anomaly go through their reproductive carrier

UN eventfully with diagnosis being made incidentally in some cases [4] . The investigative modalities for making the diagnosis are usually ultrasonography, hysterosalpingography, magnetic resonance imaging and laparoscopy. Because of the rarity of this condition and the fact that when pregnancies occur, they are fraught with complications [1-3], we considered it worthwhile to report this patient who successfully carried her pregnancy to 38 weeks gestation and had a good pregnancy outcome.

\section{CASE DETAILS}

A 22 year old primigravida presented at 20 weeks gestation for antenatal care to a rural tertiary care centre. She had no complaints but had indicated that an earlier ultrasound scan done at 11 weeks of gestation by a private practioner detected an abnormal uterus with two horns (bicornuate uterus).There were no significant findings on examination and the vulva and vagina appeared normal with a single cervix seen on speculum examination. She had regular antenatal follow-up visits and was found to have persistent breech presentation in the third trimester. The liquor was reduced and the foetus had intrauterine growth restriction. She was admitted in the antenatal wards and was kept under supervision. Aminoinfusion and intravenous fluid therapy was given. Antepartum fetal monitoring was done in the form of biophysical profile and Doppler scan. The pregnancy was carried till 38 weeks of gestation and an elective caesarean section was performed in view of bicornuate uterus and persistent breech presentation and a live male baby, weighing $2126 \mathrm{gms}$, was extracted out by breech. However due to the abnormal shape of the uterus an inverted $\mathrm{T}$ shaped incision had to be given on the uterus to deliver the baby. The baby cried immediately after delivery and was in a good condition with an Apgar score of 9/10. Placenta with membranes was delivered. On examination of placenta one lobe was missing and was found impacted in the right horn of the uterus, which was carefully removed along with all membranes. Uterus was exteriorized and on examination the uterus was almost heart shaped with two separate well developed horns separated by a septum inside, bilateral tubes and ovaries were normal, each attached to the two horns of uterus. Uterus was closed and complete haemostasis was achieved. Postoperative period was uneventful and motherbaby pair was discharged under satisfactory condition.

\section{DISCUSSION}

Incomplete fusion of the Mullerian or Paramesonephric ducts result in the most common types of uterine malformations. Two processes that occur during the embryonic development of the Mullerian ducts fusion and resorbtion - can be affected to different degrees. The degree can determine the likeliness of a pregnancy reaching full term. 
Bicornuate uterus is a congenital uterine anomaly that results from defective lateral fusion of the paramesonephric ducts at about the tenth week of intrauterine life around the fundus. As a result of this, there are two separate but communicating endometrial cavities and a single uterine cervix. Several adverse reproductive outcomes have been reported with this abnormality including breech presentation [1-3]. In this report persistent breech presentation in the third trimester was not surprising as the abnormal uterus would not provide enough room for a rotation to a cephalic position once the fetus assumed this malpresentation in the third trimester. This condition can be identified on first trimester scan thus highlighting the importance of an early ultrasonography, in addition to its other known benefits like accurate dating and determination of fetal viability. Three-dimensional ultrasonography, where available, also helps to circumvent this difficulty [2]. A renewed awareness to this rare but important condition is needed for capacity building to help make a diagnosis in order to improve understanding of its effect in pregnancy. Skilled Obstetrician is required to deliver the baby during cesarean section.

\section{CONCLUSION}

Early diagnosis and proper antenatal care is required to successfully manage a pregnancy with bicornuate uterus. Reduced liquor may increase the difficulties while delivering the baby at cesarean section. Anticipation and preparedness to deal with these known complications will ensure positive outcome for the mother and baby.

\section{REFERENCES}

[1]. Zlopasa G, Skrablin S, Kalafatic D et al. Uterine anomalies and pregnancy outcome following resectoscope metroplasty. Int $J$ Gynaecol Obstet, 2007; 98: 129-133

[2]. Salim R, Jurkoric D. Assessing congential uterine anomalies: the role of three dimensioned ultrasonography. Best Pract Res Clin Obstet Gynaecol, 2004; 1829-36

[3]. Hua M, Odibo A, Longman R, et al. Congenital uterine anomalies and adverse pregnancy outcome. A J Obstet Gynecol, 2011; 204(1): 334-335

[4]. Simon C, Martinex I, Pardo F, Tartajada M, Lellicor A. Mullerian defects in women with normal reproductive outcome. Fertil Steril 1991; 561192-3.

[5]. Nahum G G. Uterine anomalies; how common are they and what is their distribution and subtypes? J Reprod Med, 1998;43:877

[6]. Imo AOC, Sunday-Adeoye I. Radiological Assessment of the uterus and fallopian tubes in infertile women at Abakiliki Nigeria. Nigeria J Clin Pract, 2008;11: 211-215.

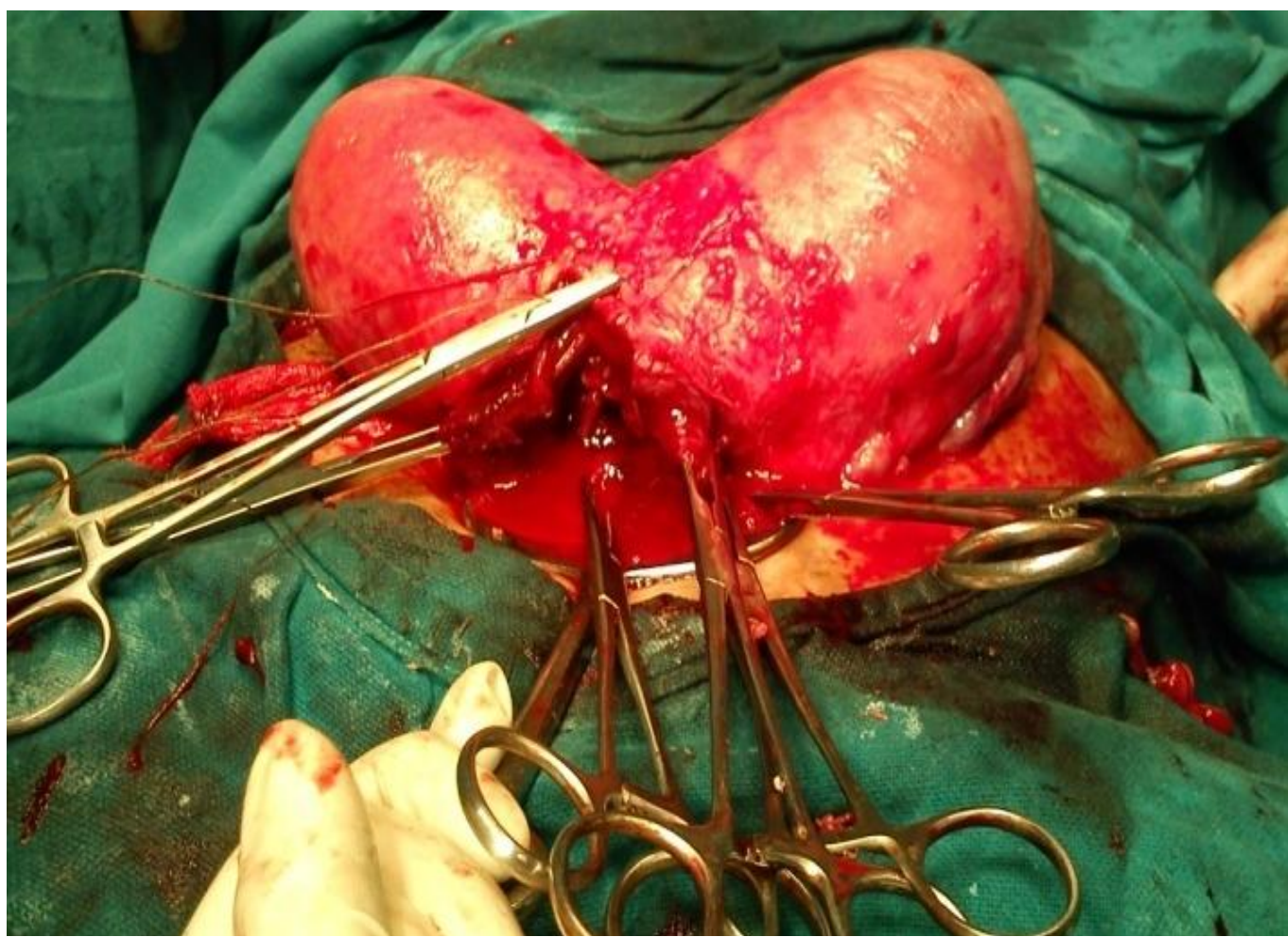

Figure1: showing bicornuate uterus with inverted $\mathrm{T}$ incision given to deliver the baby. 


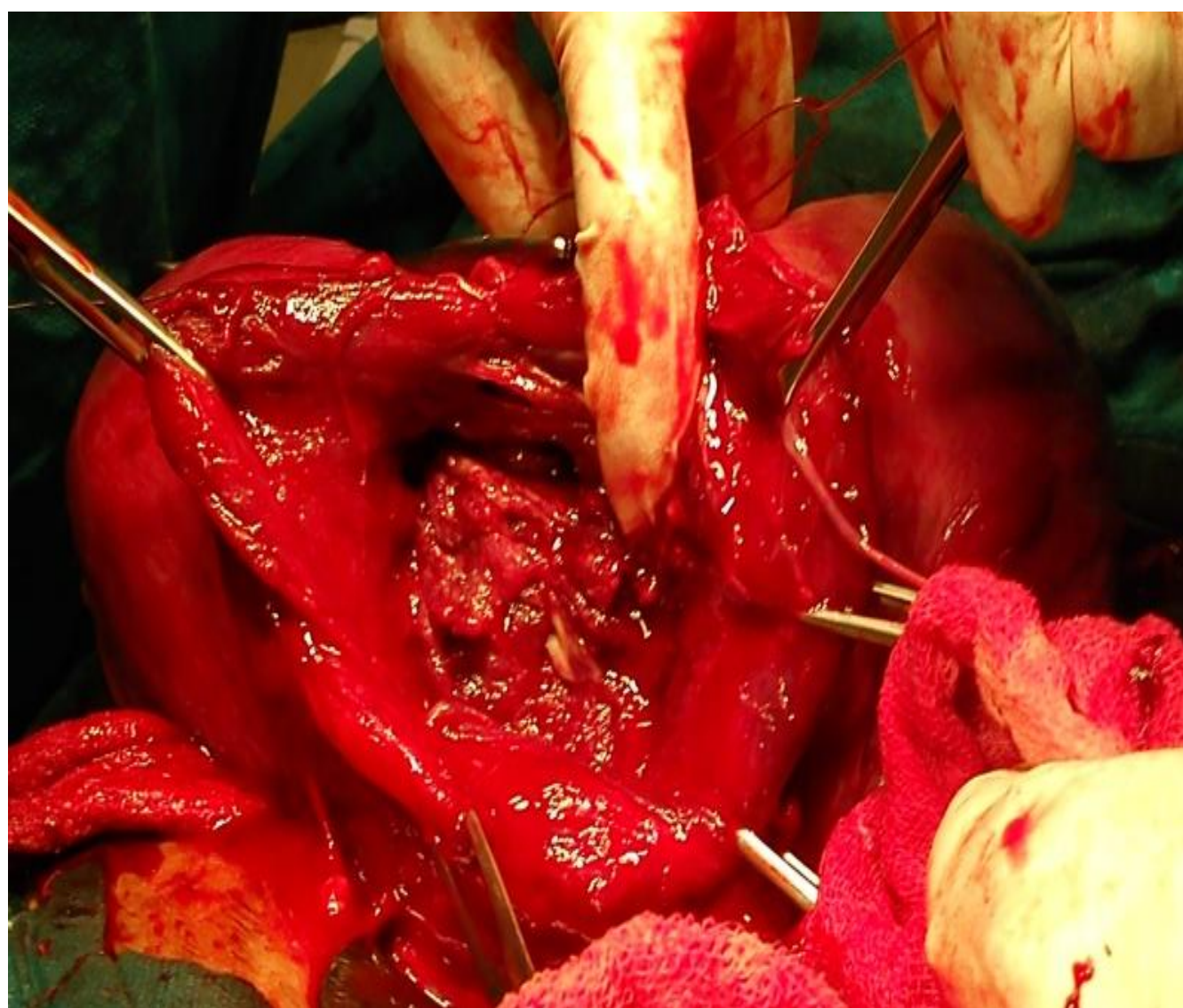

Figure2: showing the septum separating the two horns. Right horn contained the pregnancy.

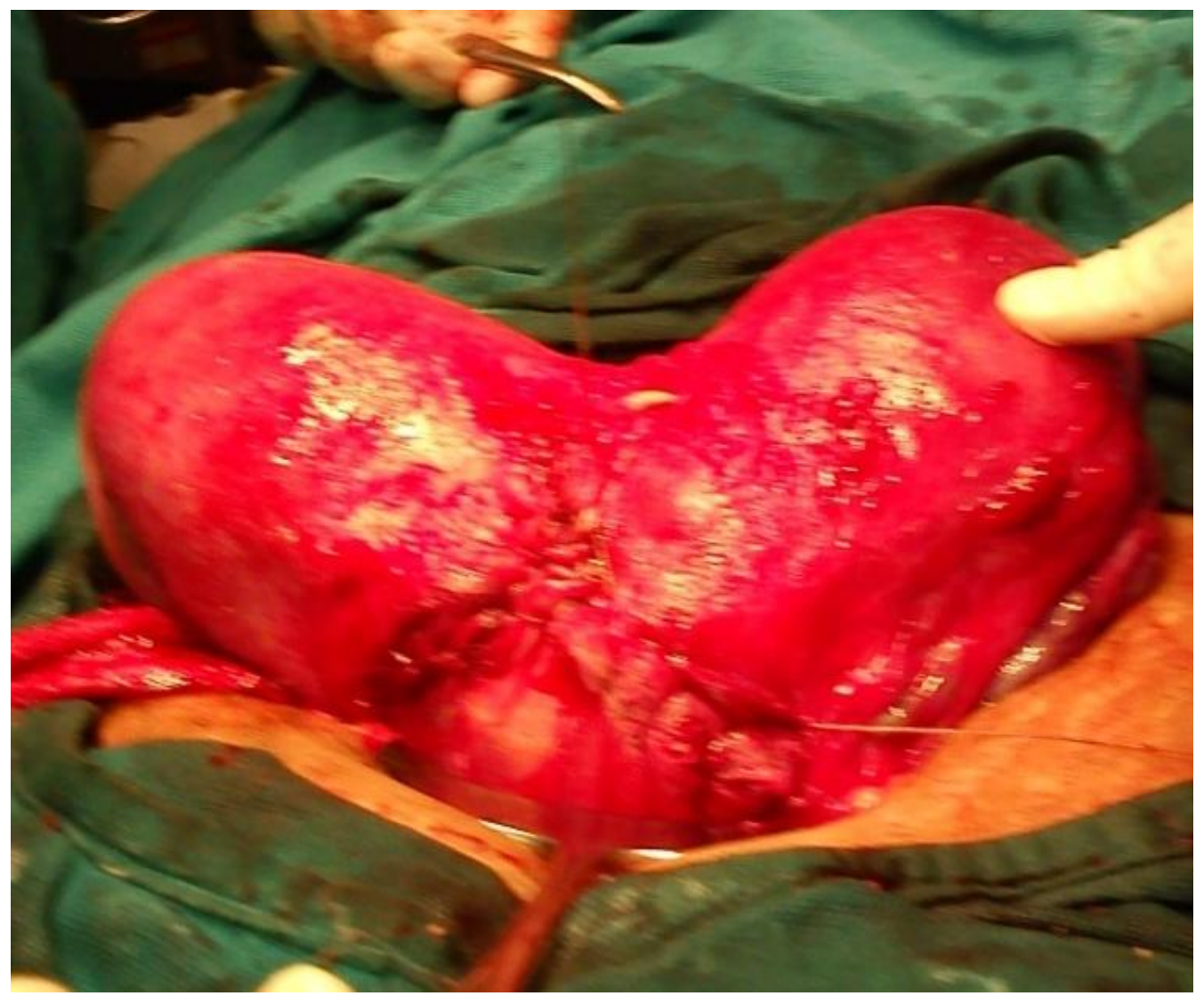

Figure3: showing the almost heart shaped uterus sutured back with complete haemostasis achieved. 